\title{
Visual understanding of divergence and curl: Visual cues promote better learning
}

\author{
Pascal Klein, ${ }^{1, *}$ Jouni Viiri, ${ }^{2}$ and Jochen Kuhn ${ }^{1}$ \\ ${ }^{1}$ Department of Physics / Physics Education Research Group, \\ Technische Universität Kaiserslautern, Erwin-Schroednger-Str. 46, 67663 Kaiserslautern, Germany \\ ${ }^{2}$ Department of Teacher Education, University of Jyväskylä, 40014 Jyväskylä, Finland
}

\begin{abstract}
Prior research has shown that students struggle to indicate whether vector field plots have zero or non-zero curl or divergence. In an instruction-based eye-tracking study, we investigated whether visual cues (VC) provided in the vector field plot can foster students' understanding of these concepts. The VC were only present during instruction and highlighted conceptual information about vector decomposition and partial derivatives.

Thirty-two physics majors were assigned to two groups, one was instructed with VC about the problemsolving strategy, and one without. The results show that students in VC-condition performed better, responded with higher confidence, experienced less mental effort, and rated the instructional quality better than students instructed without cues. All results were statistically significant. Furthermore, VC-students performed better on a transfer task about the curl concept. The superior performance of students in VC-condition can be attributed to saccadic eye-movements which are in line by correct application of the visual strategy and which were supported by the visual cues. The outcomes strongly confirm multimedia design principles and reveal a direct link between processing explicit instructions and its application in subsequent tasks in the domain of problem solving.
\end{abstract}

\section{INTRODUCTION}

Many problems in upper division physics and other scientific disciplines require students to relate abstract concepts to multiple external representations, including diagrams, equations, graphics or data tables. It is well known that visual representations of abstract concepts have the potential to substantially promote learning [1]. In a more general form, the ability to relate mathematical concepts to various representations contributes to representational competence, that is, being able to use multiple representations for understanding [2]. However, the acquisition of this skill requires instructional support, especially when concepts become more sophisticated.

One example which has recently been studied consists of the visual interpretation of two-dimensional vector field plots with respect to divergence or curl [3-7]. In simplified cases, students must decide whether vector fields have zero or nonzero curl or divergence, cf. Fig. 1. This task is challenging even for graduate students [3] and requires the interpretation of the mathematical definition of divergence and curl and its application to the vector field. In this contribution, we solely focus on the differential representation of divergence and curl in Cartesian coordinates [8], i.e.,

$$
\begin{aligned}
\operatorname{div} \vec{F}(\vec{r}) & =\vec{\nabla} \cdot \vec{F}=\frac{\partial F_{x}}{\partial x}+\frac{\partial F_{y}}{\partial y}, \\
\operatorname{curl} \vec{F}(\vec{r}) & =\vec{\nabla} \times \vec{F}=\left(\frac{\partial F_{y}}{\partial x}-\frac{\partial F_{x}}{\partial y}\right) \hat{e}_{z},
\end{aligned}
$$

where $\vec{F}=\left(F_{x}, F_{y}, 0\right), \vec{r}=(x, y, z)$, and $\vec{\nabla}$ represents the nabla (del) operator. By definition, the divergence and curl of a vector field can be evaluated qualitatively by interpreting

\footnotetext{
* Correspondence should be addressed to pklein@ physik.uni-kl.de
}

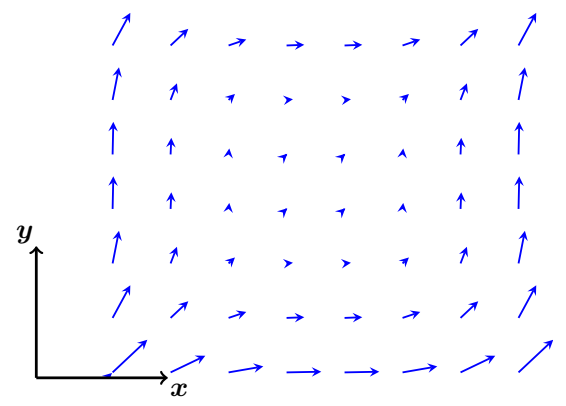

FIG. 1. Graphical representation of a two-dimensional vector field $\vec{F}(\vec{r})$ with constant divergence. Can you judge whether divergence is zero or non-zero?

the partial vector derivatives. Application to a graphical vector field plot means that one must inspect the change of both field components in the $x$ - and $y$-directions, respectively, cf. Fig. 2. To keep it simple, we only distinguish between zero and non-zero divergence and exclude cases in which two nonzero addents cancel each other out $\left(\partial F_{x} / \partial x=-\partial F_{y} / \partial y\right)$. Vividly speaking, we must perform horizontal and vertical eye movements to judge the change of the vector field in horizontal and vertical directions, respectively, making this visual task perfectly suitable for eye-tracking methodology (the saccadic direction analysis was introduced in [7]).

Prior research has shown that student's performance on this problem - even after explicit instruction -was mediocre (66\% success rate while chance of guessing correct is $50 \%$ ), and students mainly struggled with the concept of partial vector derivatives [9]. Based on previous work [6, 7], we designed a problem-solving instruction which highlights conceptual features in the vector field diagram that are important for problem-solving. We established a strong integration between written text and the field plot. In this contribution, we 


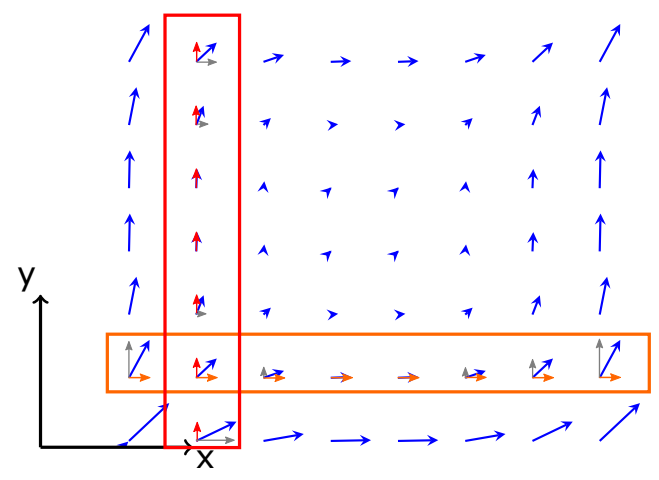

FIG. 2. Visual cues highlighting the vector field decomposition and framing one row/column for evaluating $\partial / \partial x$ and $\partial / \partial y$. For instance, the divergence can be estimated by judging the change of the orange arrows in the orange box and the red arrows in the red box, cf. Eq. (1). Remember that the fields are constructed to have constant zero or non-zero divergence everywhere.

investigate the impact of the instruction on learning, focusing on the effect of visual cuing.

\section{THEORETICAL BACKGROUND: VISUAL CUING}

Constructing a visual understanding of divergence/curl involves coordinating information provided in multiple representations with prior knowledge. We refer to the cognitive theory of multimedia learning (CTML, [10]) which helps us interpret the functions and mechanisms of constructing knowledge with multiple representations [11]. CTML identifies three distinct processes (selection, organization, and integration) involved in learning from information presented in multiple modalities. It has been argued that highlighting relevant information (visual cueing) can facilitate all of these three processes $[12,13]$ :

Selection can be described as the process of accessing pieces of sensory information from each representation. By highlighting relevant information in a visual representation, such as the vector decomposition, it is easier for the learner to attend to that information.

Organization describes the structuring of the selected information to build a coherent internal representation, involving, for example, comparisons and classifications. Highlighting relevant information emphasizes the structure and the identification of links between elements. In our example, the $x$ - and $y$-directions are highlighted with framing boxes.

Integration can be considered as combining internal representations with activated prior knowledge (long-term memory). In particular, learners need to integrate elements within a single representation or across multiple representations, for example, coordinating graphical representations and equations with text to create a mental model to solve a problem. In the present example, we used colored elements to refer to the corresponding parts in the definition of the concept.

\section{INSTRUCTION-BASED EYE-TRACKING STUDY}

\section{A. Hypotheses and research questions}

Based on the theoretical framework above, we hypothesize that problem solving abilities will be improved by the cues shown in Fig. 2 since they make implicit causal or functional relations between elements more explicit. We also investigate the impact of the instructions on mental stress and quality of instruction judgements.

H1 Instructions about the visual interpretation of divergence are more successful (in terms of performance and confidence measures) with visual cues than without. In other words, students instructed with visual cues (VC-students) will perform better in subsequent problem solving than students instructed with no cues (NC-students).

$\mathrm{H} 2 \mathrm{VC}$-students will perform better in a transfer task (interpretation of curl) than NC-students.

RQ1 What between-group differences, if any, are there concerning perceived mental effort and perceived quality of instruction?

RQ2 How does students' visual attention and saccadic eye movement differ during (a) processing of the instruction and (b) problem solving.

\section{B. Sample}

A total of 32 (26 male) participants with ages ranging from 18-27 (mean 20.6 years) took part in the experiment. All of them attended an introductory electromagnetism $(\mathrm{E} \& \mathrm{M})$ course and had successfully completed two mechanics courses (calculus-based mechanics and experimental physics). The lectures have introduced the concepts of divergence and curl traditionally without emphasizing visual interpretation. Participation was voluntary, took $30 \mathrm{~min}$, and was compensated with 10 EUR.

\section{Study Design and measures}

First, students completed a pre-test about their conceptual knowledge of divergence and curl (questions similar to those in [4]). Then, they were randomly assigned to one of two groups, the visual cue (VC)-condition and the no cue (NC)condition (16 vs. 16 participants), and conducted the experiment in front of a computer screen. The instruction page explained how the divergence of vector fields can be interpreted qualitatively. The page included (i) the formal definition of divergence, (ii) written text about the visual interpretation of the equation (problem-solving strategy), (iii) one vector field plot with or without visual cues (VC or NC) and 
(iv) some hints about the experimental procedure. The contrast between the groups is actually represented in the Figures 1 and 2. In the VC-condition, the text (ii) refers to the orange box and the red box in the diagram, and the vector decomposition shown there (small red and orange arrows). Students could take as much time as needed before proceeding to the problem-solving phase. Then they were asked to judge the divergence of eight vector field plots and rate the confidence of their judgment on a Likert scale. After responding to all eight problems, students rated the quality of the instruction (6 Likert items) as well as the mental stress during problem solving (5 Likert items) [14]. After completing the divergence problems, students were provided with the formal definition of the curl operator but received no explicit instruction. The sequence of the same vector fields followed, and students had to judge the curl of each vector field and rate their confidence.

In summary, we obtained the following measures (abbreviations in parentheses): Pre-test scores concerning divergence $\left(\mathrm{DIV}_{\text {pre }}\right)$ and curl concepts $\left(\mathrm{CURL}_{\text {pre }}\right)$, performance scores after instruction (DIV and CURL), confidence scores (DIV conf. and CURL $_{\text {conf. }}$, perceived quality of instruction (QI), and perceived mental stress (MS).

\section{Eye-tracking procedure and data analysis}

We obtained gaze data for all students using a Tobii X3120 eye-tracker installed on a 24" LCD screen. The device has an accuracy of 0.4 degrees and allows a relatively high freedom of head movement. Gaze data was recorded using the Tobii Studio Pro software.

In this contribution, we make use of heatmaps, showing how visual attention is distributed over an area for each group of students. Furthermore, we divided the instruction page into four areas of interest (AOI) i) - iv) as described in Sect. III C, see also Fig. 3 (a), and calculated average dwell time on each AOI. Last, we used saccadic direction analysis (SDA) to obtain information about the distribution of saccadic angles. As explained above, correct evaluation of the partial derivatives requires vertical and horizontal eye movements, hence SDA is a useful tool for this particular task.

\section{RESULTS}

\section{A. Descriptive statistics (H1, H2, and RQ1)}

Table I presents the average values of students' performance, confidence, mental stress, and perceived quality of instruction measures by group. All values have a possible range between 0 and 1 , where 1 represents perfect performance, highest confidence, high mental stress, and high quality of instruction, respectively. Criteria to treat the data sets as sufficiently normally distributed were met. As can be seen, there was no significant difference regarding prior knowledge
TABLE I. Means and standard errors in parentheses. Statistics refer to a $t$-test. Effect size measure is Cohen's $d$.

\begin{tabular}{lccccc}
\hline & No. of & visual cues & no cues & \multicolumn{2}{c}{ statistics } \\
measures & items & $(N=16)$ & $(N=16)$ & $p$ & $|d|$ \\
\hline DIV $_{\text {pre }}$ & 9 & $0.75(0.04)$ & $0.70(0.04)$ & 0.55 & $\ldots$ \\
CURL $_{\text {pre }}$ & 8 & $0.76(0.04)$ & $0.68(0.04)$ & 0.20 & $\ldots$ \\
DIV & 8 & $0.82(0.03)$ & $0.71(0.04)$ & 0.04 & 0.37 \\
DIV $_{\text {conf }}$ & 8 & $0.74(0.02)$ & $0.53(0.02)$ & $<0.001$ & 1.28 \\
CURL & 8 & $0.76(0.04)$ & $0.61(0.04)$ & 0.01 & 0.46 \\
CURL $_{\text {conf }}$ & 8 & $0.73(0.02)$ & $0.56(0.02)$ & $<0.001$ & 0.97 \\
MS & 5 & $0.27(0.02)$ & $0.38(0.03)$ & $<0.001$ & 0.70 \\
QI & 6 & $0.81(0.02)$ & $0.61(0.03)$ & $<0.001$ & 1.22 \\
\hline
\end{tabular}

Note: Abbreviations of measures are explained in the text.

between groups. Students instructed with visual cues performed better on the divergence problems and on the transfer problems regarding curl. Both effects are significant and medium-sized [15]. These students replied with much more confidence, resulting in a large between-group effect. Moreover, VC-students experienced less mental stress (mediumsized effect) and judged the quality of instruction better.

\section{B. Eye-Tracking data (RQ2)}

The heatmaps shown in Fig. 3 reveal that students without visual cues paid less attention on the field plot than students instructed with the cues. In numbers, the visit duration on the $\mathrm{AOI}_{\text {iii }}$ is $25.2 \mathrm{~s}$ in the $\mathrm{VC}$-condition in contrast to $7.81 \mathrm{~s}$ in the NC-condition ( $p=0.04, d=0.74)$. Total time spent on the instruction page was $102.71 \mathrm{~s}$ (no between-group effect).

Furthermore, we applied saccadic direction analysis [7] on the eye movement data. Considering the field plot presented in the instruction, we identify that VC-students performed dominantly horizontal and vertical eye-movements, see Fig. 3 (c). In contrast, the distribution of NC-students is more broad and contains oblique saccades. This difference can also be observed during problem solving.

In Fig. 4, we show item number 2 and the gaze path of one VC- and one NC-student. This vector field has only one nonzero component in $y$-direction, hence we expect dominantly vertical saccades. The student who correctly concluded that the divergence of this field is zero (blue circles) proceeded systematically and checked one column of arrows in vertical direction. He also checked one row in horizontal direction. The other student (orange circles) who failed in this task performed dominantly horizontal saccades and checked several rows of arrows. The figure also shows students' saccadic direction distribution as a function of correct or incorrect answers. We observe differences similar to the results of the instructional field, viz., correct application of the visual strategy implies concentrating on vertical and horizontal directions. 


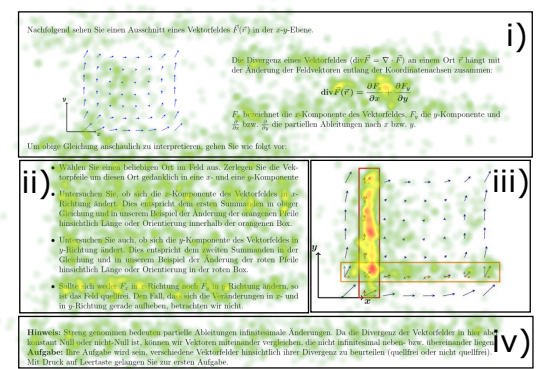

(a)

(b)
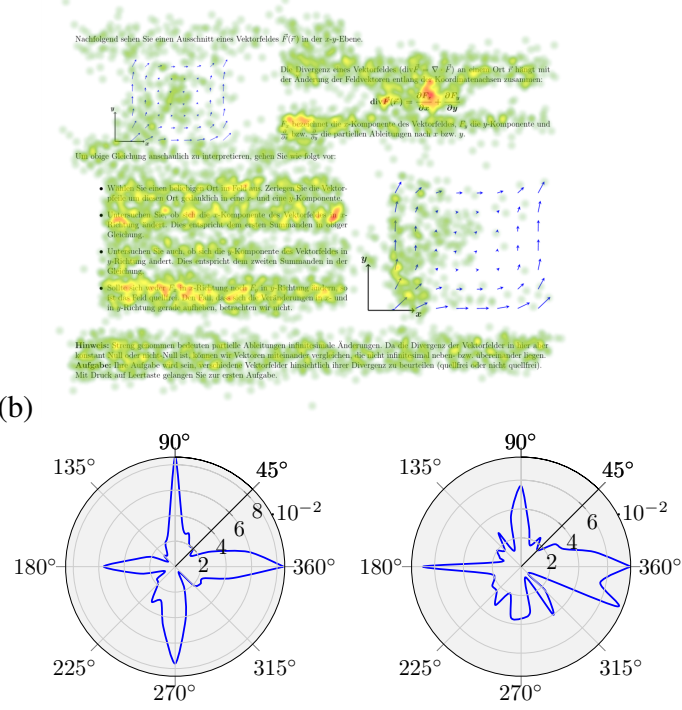

(c)

FIG. 3. Students' visual attention on the (a) VC- and (b) NCinstruction with definition of AOIs i)-iv). (c) Saccadic direction distribution of VC-students (left) and NC-students (right) when they observed the vector field plot on the instruction page (part iii).

\section{CONCLUSION}

Even though the experimental condition included only a minor change (adding visual cues and emphasize the textgraph-connection), the effect on learning was immense. The results confirm design principles of multimedia learning, that is, providing graphical representations with visual cues (VC) improves learning. The instruction with conceptual highlights included important information about vector decom- position and spatial information about partial derivativesconcepts that are crucial in the subject context. Discrepancies between both groups concerning mental stress, confidence in students' answers as well as perceived instructional quality emphasize the positive impact of VC. Conversely, the results also show that vector decomposition and partial derivatives can still pose serious difficulties to end-of-first-year students.

Eye-tracking data strongly indicate that visual cues are helpful to shift the focus of attention to the relevant parts of the content and to put emphasis on the conceptual relations between the elements. Furthermore, we observed that students processed the vector field during instruction differently between groups. VC-students performed dominantly horizontal and vertical eye movements, reflecting correct interpretation of the partial derivatives. These eye gaze patterns were also found during successful problem solving.

Please note that all vector fields used in this study had either zero or non-zero divergence everywhere, what might reinforcing a common misconception that a field has only a single value for divergence rather than divergence being a local property of the field. To avoid this misconception, students were told about this special case in the instruction (part iv).

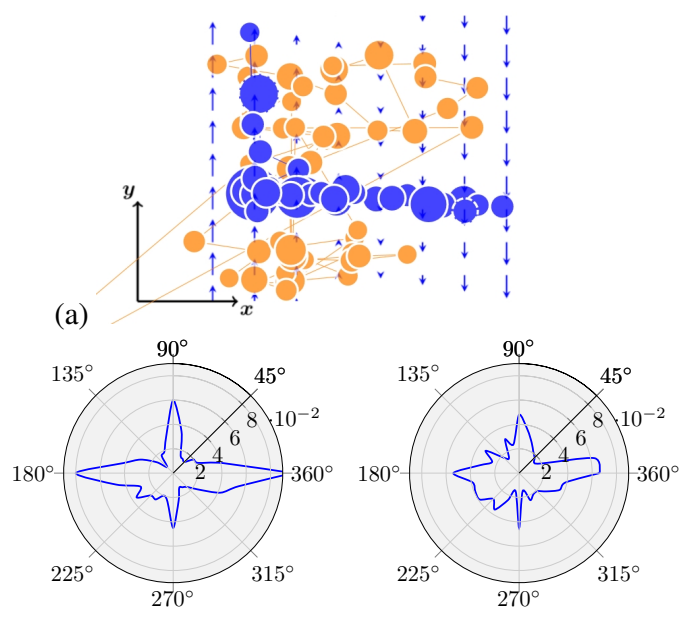

FIG. 4. (a) Gaze plots (sequence of fixations) of students judging the divergence of a vector field correctly (blue, VC) and incorrectly (orange, NC). b) Saccadic direction distributions concerning the same vector field of all students answering correct (left) or incorrect.
[1] D.E. Meltzer, Am. J. Phys. 73, 463 (2005)

[2] M. De Cock, Phys. Rev. Phys. Educ. Res. 8(2), 2012.

[3] C. Singh and A. Maries, AIP Conf. Proc. 1513, 382 (2013).

[4] C.R. Baily et al., PER Conference series 2016, pp. 51-54.

[5] L. Bollen et al., Phys. Rev. Phys. Educ. Res. 12, 020134 (2016).

[6] P. Klein and J. Kuhn, PER Conference series 2017, pp. 220-3.

[7] P. Klein et al., Phys. Rev. Phys. Educ. Res. 14, 010116 (2018).

[8] Integral representations of divergence are discussed in [6, 7].

[9] R. Pepper et al., Phys. Rev. Phys. Educ. Res. 8, 010111 (2012).

[10] R. E. Mayer, Multimedia learning (2009).
[11] S. Ainsworth, Comput. Educ. 33, 131 (1999).

[12] B. B. de Koning et al., Educ. Psychol. 21, 113 (2009).

[13] Madsen et al., Phys. Rev. Phys. Educ. Res. 9, 020104 (2015).

[14] All items were created by the first author. Example: (MS2) Vector decomposition was difficult, (MS3) Judging the change of components was difficult. (QI2) Essential steps were provided. (QI3) The instruction was well structured.

[15] J. Cohen, Curr. Dir. Psychol. 1, 98 (1992). 